\title{
ANÁLISE DE TÉCNICAS DE RECONCILIAÇÃO DE DADOS APLICADAS A UM SISTEMA VASO SEPARADOR
}

\author{
Felipe Santana Santos* Leocarlos Bezerra da Silva Lima* \\ *Av. Mal. Rondon, $s / n$ \\ Cidade Universitária Prof. José Aloúsio de Campos \\ 49100-000, São Cristóvão-SE, Brasil
}

Email: felipesotransacoes@gmail.com leocarlos@ufs.br

\begin{abstract}
The measurement of variables from an industrial process is of great importance in systems in which decisions are made based on these measurements, as in control systems. However, these measurements are always accompanied by errors, random or gross. This is not different in the process of oil separating. An alternative for a greater reliability of the variables is the use of data reconciliation (DR). RD arises from the development of techniques for measurement and data storage. Its role is to ensure the consistency of data using the redundancy of the measurements and a statistical model of the measurements to increase its accuracy. This work investigates techniques for data reconciliation and its use in reducing the errors observed in measurements on a separator vessel.
\end{abstract}

Keywords - Random erros, data reconciliation, separator vessel.

Resumo- A medição das variáveis envolvidas em um processo industrial tem uma grande importância em sistemas em que decisões são tomadas baseadas nessas medições, como é o caso de sistemas de controle. Porém, essas medições sempre são acompanhadas de erros, sejam eles aleatórios ou grosseiros. No processo de separação do petróleo utilizando o sistema vaso separador isso não é diferente. Uma alternativa para uma maior confiabilidade das variáveis é o uso da reconciliação de dados (RD). A RD trata de um problema advindo da evolução das técnicas de medição e armazenamento de dados. Esta tem o papel de garantir a consistência destes dados, utilizando a redundância das variáveis medidas e um modelo estatístico da medição para aumentar a precisão dos dados. Este trabalho investiga as técnicas de reconciliação de dados e seu emprego na redução dos erros observados nas medições em um sistema vaso separador.

Palavras-chave- Erros aleatórios, reconciliação de dados, vaso separador.

\section{Introdução}

Para um bom funcionamento de qualquer sistema automatizado, seja estacionário ou dinâmico, na industria química ou em sistemas robóticos, é imprescindível o emprego de um controlador baseado em modelo adequado do processo, e alimentado com medições confiáveis das variáveis de estado de interesse (Freire et al., 2008).

Os dados de um processo são obtidos através de instrumentos físicos e rotinas de medição de precisão finita. Em geral, erros de medição podem ser categorizados em duas classes (Benqlilou, 2004):

1. Erros aleatórios: devidos a variações ou distúrbios de natureza não controlada. Ex.: ruído. São considerados independentes e com distribuição normal de média zero;

2. Erros não aleatórios ou grosseiros: são gerados por eventos não aleatórios, podendo estar relacionados com a medição (mal funcionamento dos instrumentos, por exemplo) ou com o processo (vazamentos, perdas, modelos inadequados ou imprecisos, etc.).

O emprego de dados corrompidos pode comprometer a atuação do controle do processo, podendo levar uma planta a operar num ponto subótimo ou inseguro, levar a perda de especifica- ção de produtos, poluição ambiental, perdas financeiras ou altos custos de operação (Morad et al., 2005).

A chamada reconciliação de dados (RD) é uma técnica voltada à melhoria da confiabilidade das medições realizadas e constitui abordagem de filtragem baseada em modelos do sistema (Bai et al., 2006; Crowe, 1996; Derya B. Özyurt, 2004; Tjoa and Biegler, 1991; Darouch and Zasadzinski, 1991; Kuhen and Davidson, 1961). As técnicas de RD baseiam-se na redundância das medidas e em restrições impostas por modelos do sistema. Dependendo das restrições do modelo do processo, a $\mathrm{RD}$ pode ser estacionária ou dinâmica, como também linear ou não linear (Benqlilou, 2004). Ela pode possibilitar a redução da frequência de calibração de sensores e a diminuição de erros nas medições, tornando o monitoramento de plantas mais preciso para tomada de decisão (Lawrence, 1989).

Objetiva-se aqui analisar as técnicas de reconciliação de dados e seu emprego na redução dos erros observados nas medições em um sistema vaso separador (Nunes et al., 2010).

A próxima seção descreve as técnicas de RD. A seção 3 descreve o sistema vaso separador. $\mathrm{Na}$ seção 4 apresenta os resultados obtidos em simulação para as técnicas abordadas. Na sexta seção, são descritas as conclusões do trabalho. 


\section{Reconciliação de Dados}

Erros aleatórios são causados por ruído, portanto não podem ser totalmente eliminados. Considerando que estes erros são provenientes de um número suficientemente grande de diferentes fontes, o teorema central do limite aplica-se (Bagajewicz and Jiang, 1997) e a soma destes distúrbios tende à distribuição gaussiana. Assim, erro aleatórios são modelados como ruído branco gaussiano $N\left(0, \sigma^{2}\right)$.

Erros grosseiros geralmente são decorrentes de possíveis falhas num determinado equipamento de medição. Tais erros também podem ser considerados com função densidade de probabilidade gaussiana, porém com média diferente de zero $N\left(\beta, \sigma^{2}\right)$ (Tjoa and Biegler, 1991). Por este motivo, o efeito prejudicial deste tipo de erro é muito maior do que o do aleatório.

O problema de RD pode ser visto como um problema de minimização do erro médio quadrático entre as variáveis medidas e as descritas por um modelo (Farias, 2009). Este problema tem como restrições modelos do processo que podem ser: lineares, bilineares ou não-lineares, assim como estáticos ou dinâmicos. As técnicas de RD foram criadas baseadas nestas restrições e podem ser divididas em dois grandes grupos: reconciliação de dados estacionária (RDE) e reconciliação de dados dinâmica (RDD).

O conceito de RD foi inicialmente proposto por Kuhen and Davidson (1961) para calcular balanços de massa em estado estacionário. A solução proposta por Kuhen and Davidson (1961) para a resolução deste problema foi obtida empregando multiplicadores de Lagrange, sendo dada por

$$
\hat{\mathbf{y}}=\mathbf{y}-\mathbf{V A}^{T}\left(\mathbf{A V A}^{T}\right)^{-1} \mathbf{A y}
$$

em que $\mathbf{V}$ é a matriz de covariâncias, y é o vetor das variáveis medidas, $\hat{\mathbf{y}}$ é o vetor das estimativas reconciliadas das variáveis medidas e $\mathbf{A}$ é a matriz de restrições lineares.

Sistemas dinâmicos são representados por equações diferenciais que descrevem a dependência das variáveis do processo no tempo. De modo geral, sistemas dinâmicos são representados por equações algébrico-diferenciais, com restrições não lineares e muitas vezes com parâmetros e condições iniciais desconhecidas (Prata, 2009). Por isso, os problemas de RDD são mais complexos que os de RDE, porém a RDD apresenta um número maior de aplicações na indústria, em que os processos estão frequentemente variando suas características.

Gertler and Almasy (1973) foram dos primeiros a estudar RDD. Eles estudaram modelos dinâmicos de balanço material representados por equações de estado contínuas. Stanley and Mah (1977) propuseram o uso de filtro de Kalman (FK) como solução do problema de RDD. Essa técnica iterativa é útil em modelos dinâmicos lineares para reconciliar e/ou estimar dados. Ela exige que o erro tenha média zero, ou seja, que não seja grosseiro.

O FK possui duas fases: predição e atualização. A fase de predição usa a estimativa do estado da iteração anterior para produzir uma estimativa do estado na iteração atual. Na fase de atualização, a medição da iteração atual e a covariância do erro são usadas para melhorar a medição e chegar a uma estimativa mais precisa do estado. Ele é descrito pelas equações

$$
\begin{aligned}
& \mathbf{X}_{t}=\mathbf{A} \mathbf{X}_{t-1}+\mathbf{B U}_{t-1}+\mathbf{W}_{t-1} \\
& \mathbf{Y}_{t}=\mathbf{C X}_{t}+\mathcal{E}_{t},
\end{aligned}
$$

em que $\mathbf{A}, \mathbf{B}$ e $\mathbf{C}$ são matrizes determinísticas com dimensões apropriadas, $\mathbf{X}_{t}$ é um vetor com os valores reais no instante de amostragem $t, \mathbf{U}_{t-1}$ é um vetor com as variáveis de entrada, $\mathbf{Y}_{t}$ é um vetor das variáveis que contêm erros, $\mathcal{E}_{t}$ é um vetor de ruído branco gaussiano de distribuição $N(0, V)$, $\mathbf{W}_{t}$ é um modelo de ruído assumido como $N(0, S)$.

O FK na forma que foi proposto inicialmente não apresenta bons resultados em sistemas não lineares. Para emprego em tais sistemas, Jang et al. (1986) propuseram o chamado filtro de Kalman estendido (FKE). Ele usa uma formulação iterativa e sequencial do problema de estimação baseada numa descrição linearizada do modelo dinâmico. O FKE apresenta desempenho melhor sob perturbação, quando comparado ao FK.

Jang et al. (1986), e posteriormente Liebman et al. (1992), propuseram o uso de uma janela móvel no tempo. Essa técnica é muito eficaz na melhoria do desempenho do controle de um determinado processo. Ela pode ser usada em vários tipos de processos, tais como: dinâmicos, contínuos, discretos, lineares e não lineares. Esta técnica apresenta muitas vantagens, dentre elas: não é necessário manter todas as informações do processo, mas apenas as do período da janela, diminuindo os requisitos computacionais. O algoritmo da técnica da janela móvel no tempo pode ser formulado pelas equações

$$
\begin{array}{r}
\min J\left(\hat{\mathbf{y}}_{t}, \hat{\mathbf{z}}_{t}\right)=\sum_{k=0}^{L-1}\left[\left(\mathbf{y}_{t-k}-\hat{\mathbf{y}}_{t}\right)^{T} \mathbf{V}^{-1}\right. \\
\left.\left(\mathbf{y}_{t-k}-\hat{\mathbf{y}}_{t}\right)+\mathbf{f}^{T}\left(\hat{\mathbf{y}}_{t}, \hat{\mathbf{z}}_{t}\right) \lambda^{-1} \mathbf{f}\left(\hat{\mathbf{y}}_{t}, \hat{\mathbf{z}}_{t}\right)\right],
\end{array}
$$

sujeito às condições

$$
\mathbf{y}_{l} \leq \hat{\mathbf{y}}_{t} \leq \mathbf{y}_{u}, \quad \mathbf{z}_{l} \leq \hat{\mathbf{z}}_{t} \leq \mathbf{z}_{u}
$$

em que $L$ é o tamanho da janela, $\hat{\mathbf{y}}$ é o vetor das variáveis reconciliadas, $\hat{\mathbf{z}}$ é o vetor das variáveis não medidas que foram estimadas, $\mathbf{V}$ é a matriz de covariância dos erros de medição, $\lambda$ é a matriz de covariância do modelo de erros aleatórios assumidos como sendo normalmente distribuídos, $\mathbf{y}_{l}$, 
$\mathbf{y}_{u}, \mathbf{z}_{l}$ e $\mathbf{z}_{u}$ são vetores de limites inferiores e superiores para as variáveis medidas e não medidas e $\mathbf{f}$ é um vetor de restrições funcionais do modelo que podem ser equações algébricas ou diferenciais.

Darouch and Zasadzinski (1991) propuseram uma técnica para a RDD, em que o modelo é dinâmico e linear e todas as variáveis são medidas. Liebman et al. (1992) propuseram um novo método capaz de reconciliar e estimar parâmetros para sistemas dinâmicos e não lineares, de estimar as variáveis de estado e de lidar com restrições de desigualdade. Albuquerque and Biegler (1996) propuseram o uso do método Runge-Kutta implícito para discretizar as equações diferenciais. Bagajewicz and Jiang (1997) propuseram um novo método para RDD em sistemas lineares.

Bai et al. (2006) propuseram um algoritmo preditor-corretor para resolver o problema da RDD. Nele, os valores das medições podem ser estimados a partir de previsões do modelo. As informações das previsões do modelo podem ser combinadas com as informações tomadas a partir das medições para dar uma melhor estimativa aos valores reais. A equação do algoritmo preditorcorretor é

$$
\hat{\mathbf{x}}_{t}=\hat{\mathbf{y}}_{t}+\mathbf{K}\left(\mathbf{y}_{t}-\hat{\mathbf{y}}_{t}\right)
$$

em que $\mathbf{K}=\left(\mathbf{I}+\mathbf{V R}^{-1}\right)^{-1}$, sendo $\mathbf{I}$ uma matriz identidade, $\mathbf{V}$ a matriz de covariância dos erros medidos, $\mathbf{R}$ a matriz de covariância dos erros preditos no modelo, $\mathbf{y}_{t}$ o vetor das variáveis medidas no processo, $\hat{\mathbf{x}}_{t}$ o vetor das melhores estimações de $\mathbf{x}_{t}$ (valores reais das medições), ou seja, o vetor das variáveis reconciliadas, e $\hat{\mathbf{y}}_{t} \mathrm{o}$ vetor de predições do modelo. K é o ganho do algoritmo preditor-corretor (varia de 0 a 1 ), que reflete o impacto do erro nas medições e nas predições do modelo quando os erros nas medições são muito maiores do que os erros de predição do modelo. Se $\mathbf{V} \gg \mathbf{R}$ e $\mathbf{K} \rightarrow 0$, consequentemente os valores reconciliados se aproximam dos valores preditos pelo modelo. Quando as predições do modelo apresentam erros muito grandes, então $\mathbf{V} \ll \mathbf{R}$ e $\mathbf{K} \rightarrow 1$, logo os dados reconciliados se aproximam dos dados contaminados pelo ruído. Observando a equação 6 nota-se que tal algoritmo é essencialmente equivalente ao $\mathrm{FK}$, diferindo apenas na informação necessária e na maneira como o ganho é calculado.

\section{Sistema Vaso Separador}

O petróleo proveniente dos poços de perfuração submarinos contém fundamentalmente óleo, gás e água, que necessitam ser separados, o que é feito pelo emprego de um vaso separador (Nunes et al., 2010; de Oliveira Azevedo JÚnior, 2009). O vaso separador é um vaso de pressão, comumente cilíndrico (horizontal ou vertical) e eventualmente esférico. Um separador trifásico é, em geral, um vaso cilíndrico e constituído por duas seções: uma câmara de separação e uma câmara de óleo. Estas são separadas por um vertedouro, denominado chicana. Os líquidos fluem na direção do vertedouro e ao longo deste caminho pratos paralelos ajudam na separação líquido-líquido. Uma fase rica em óleo transborda sobre a chicana para a câmara de óleo, então esta é transferida aos tratadores, que retiram sais e resíduos de água. O gás já separado do óleo e da água é canalizado para compressores, então este é direcionado a uma planta de tratamento, onde a umidade é retirada. A figura 1 ilustra um esquema de vaso separador trifásico horizontal.

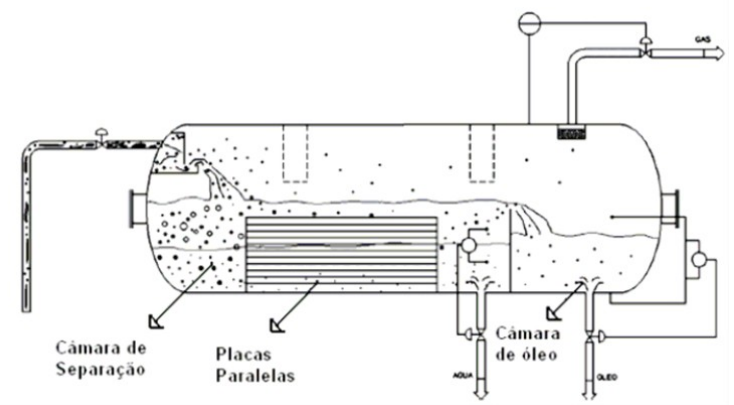

Figura 1: Vaso separador trifásico horizontal.

\section{Resultados}

As técnicas de reconciliação de dados escolhidas foram implementadas e simuladas usando Matlab/Simulink. As técnicas selecionadas para a reconciliação de dados foram o filtro de Kalman (Mah et al., 1976), um algoritmo baseado em um preditor-corretor (Bai et al., 2006) e o uso de uma janela móvel (Jang et al., 1986; Liebman et al., 1992).

Um modelo preciso do sistema vaso separador implementado em Matlab/Simulink foi utilizado. As variáveis controladas no vaso separador eram os níveis da fase oleosa na câmara de óleo, $h l$, e da interface na câmara de separação, $h w$, e a pressão de gás no vaso, $P$. As técnicas de RD foram aplicadas a duas delas, a pressão de gás $P$ e o nível de óleo $h l$.

A comparação entre as técnicas de RD foi feita com base no erro médio quadrático entre as variáveis reconciliadas e as variáveis originais (não corrompidas pelo ruído), para um mesmo nível de degradação das medições pelo ruído. Através da figura 2 , verifica-se que o comportamento da variância do erro médio quadrático (EMQ) é linear em relação ao aumento da variância do ruído presente nas variáveis. Isso implica que, independentemente da magnitude do ruído, a técnica de RD irá atenuar o mesmo de modo proporcional. Conclui-se que o EMQ constitui uma figura de 
mérito adequada para uma comparação da eficácia entre diferentes técnicas de RD na presença de ruído gaussiano.

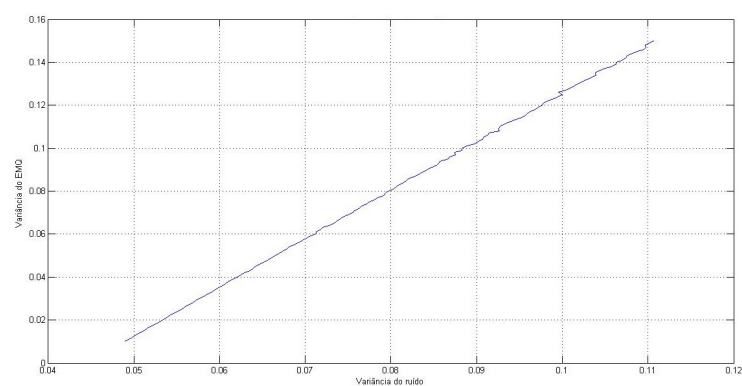

Figura 2: Variância do ruído $\times$ variância do EMQ para o FK aplicado à variável $P$.

\subsection{Filtro de Kalman}

Aplicando-se o FK nas variáveis $P$ e $h l$, obteve-se, respectivamente, $k=0,2153$ e $k=0,1333$ como ganhos do filtro. Observando a figura 3, podese notar que o FK apresentou um bom resultado em grande parte do tempo. Entre os instantes $t=750 \mathrm{~s}$ e $t=850 \mathrm{~s}$, o resultado não foi tão satisfatório em virtude das golfadas provenientes da produção de petróleo. Observa-se, então, que o FK não apresenta bons resultados na presença de perturbações (Bai and Thibault, 2010).

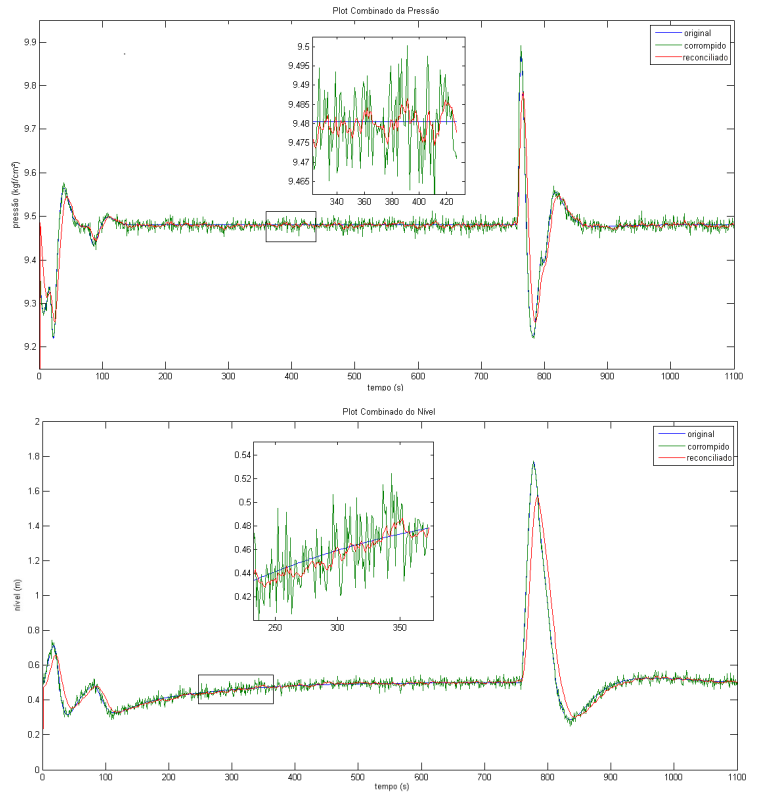

Figura 3: Variáveis $P$ e $h l$ reconciliadas empregando-se o filtro de Kalman.

Na figura 4, observa-se o EMQ das variáveis de pressão de gás e de nível de óleo ilustradas na figura 3. Como pode-se ver, no intervalo entre $t=750 \mathrm{~s}$ e $t=850 \mathrm{~s}$, o resultado não é tão bom em razão da ocorrência de perturbações. A variância do erro médio quadrático associado ao nível de óleo foi de $3,7385.10^{-4}$ e à pressão de gás foi de $6,3141.10^{-5}$.
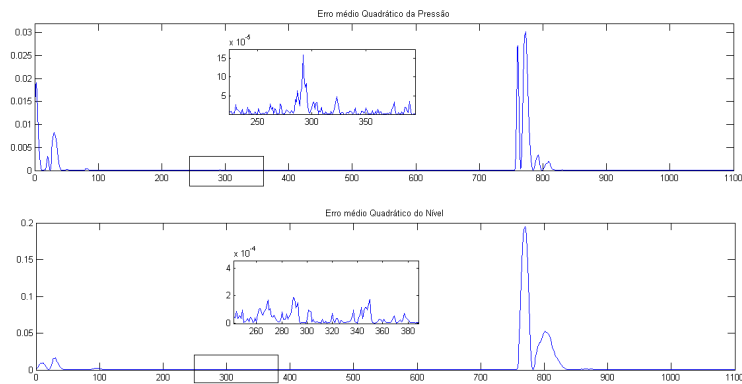

Figura 4: Erro médio quadrático associado às variáveis $P$ e $h l$ utilizando-se o FK.

\subsection{Algoritmo baseado em um preditor-corretor}

Aplicando-se o algoritmo baseado em um preditorcorretor nas variáveis $P$ e $h l$, obteve-se, respectivamente, $k=0,1962$ e $k=0,2240$ como ganhos do algoritmo. Na figura 5, observa-se que o algoritmo apresentou um bom resultado, exceto entre os instantes de tempo $t=750 \mathrm{~s}$ e $t=850 \mathrm{~s}$, em que incidem perturbações.

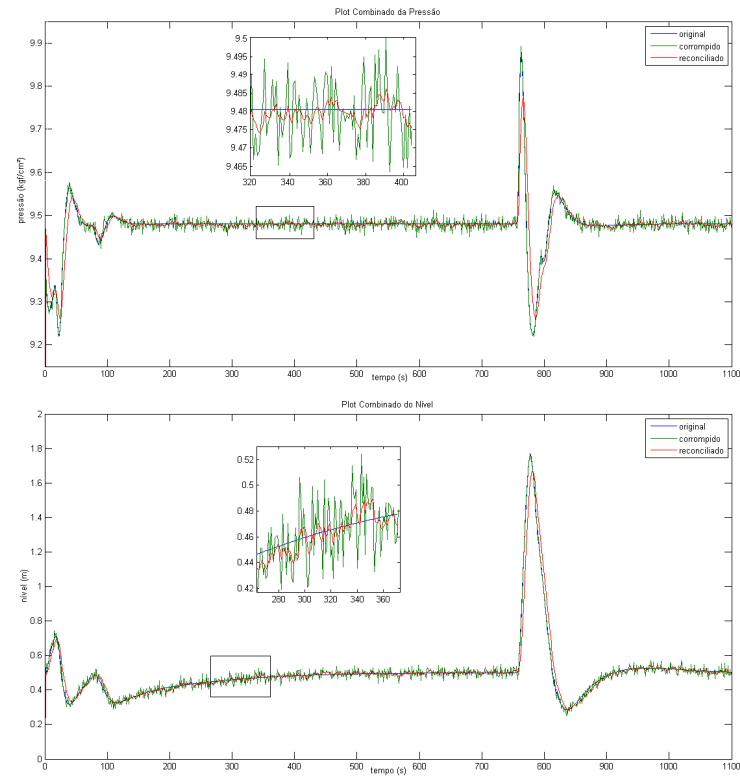

Figura 5: Variáveis $P$ e $h l$ reconciliadas empregando-se o algoritmo baseado em preditorcorretor.

A figura 6 ilustra o EMQ associado à pressão de gás e ao nível de óleo. A variância do EMQ associado ao nível de óleo foi de $5,4407.10^{-5}$ e à pressão de gás foi de $1,0969.10^{-5}$.

Da mesma forma que no caso do FK, o algoritmo baseado em preditor-corretor também apresenta melhor desempenho quando o erro presente 

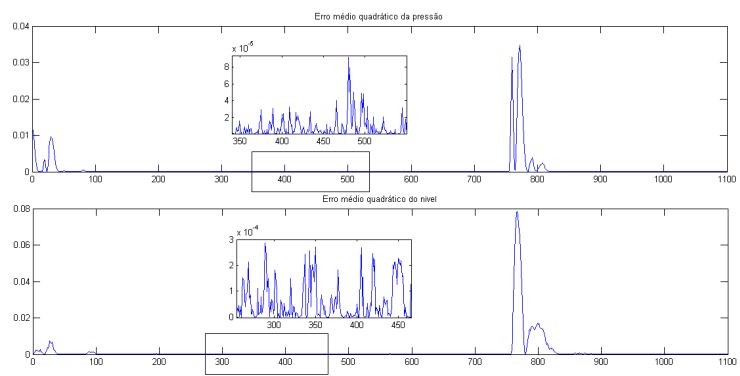

Figura 6: Erro médio quadrático associado às variáveis $P$ e $h l$ utilizando-se o algoritmo baseado em preditor-corretor.

nas medições é gaussiano (Bai et al., 2006). Também, da mesma forma que no caso do FK, esse algoritmo apresenta uma relação linear entre a variância do ruído e a variância do EMQ, comportamento ilustrado na figura 2.

\subsection{Janela móvel}

O tamanho da janela escolhida para esta abordagem algorítmica não pode nem ser muito grande, nem muito pequena. Também não há procedimento heurístico que permita a determinação do tamanho da janela a priori, sendo necessários testes de simulação para esse fim. Aqui, são apresentados os resultados para um tamanho de janela $H=250$ s, o melhor obtido no caso simulado. O ambiente simulado aqui é de 2000s, apesar dos gráficos só mostrarem um intervalo de 1100s para melhor visualização dos resultados.

$\mathrm{Na}$ figura 7, observa-se que o resultado da $\mathrm{RD}$ foi satisfatório mesmo na presença de perturbação. A variância do EMQ encontrado foi de $1,0164.10^{-7}$ para o nível de óleo e de $2,7463.10^{-8}$ para a pressão de gás. Observa-se que o uso da janela melhorou bastante a qualidade da reconciliação, pois o EMQ foi bem inferior aos obtidos nos casos dos algoritmos apresentados nas seções 4.1 e 4.2 .

\subsection{Comparação entre os métodos escolhidos}

Na tabela 1, observa-se que o uso de uma janela móvel fez com que o resultado da reconciliação fosse otimizado, quando comparado aos outros dois métodos. Esse resultado mostra que o uso de tal técnica tem um desempenho melhor na presença de perturbações. Também pode-se observar que o resultado do algoritmo baseado num preditor-corretor foi melhor do que o FK. Tal algoritmo é essencialmente equivalente ao FK, diferindo apenas na informação necessária e na maneira como os ganhos são calculados.
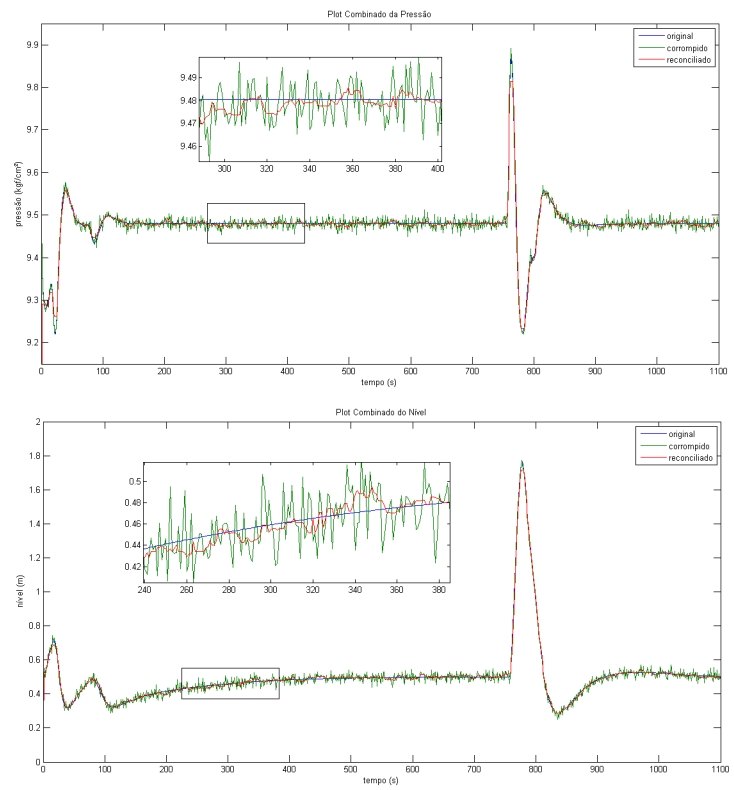

Figura 7: Variáveis $P$ e $h l$ reconciliadas empregando-se uma janela com tamanho $H=$ 250 s.

\begin{tabular}{|c||c|c|}
\hline & $\operatorname{Var}(\mathrm{EMQ} \mathrm{p} / P)$ & $\operatorname{Var}(\mathrm{EMQ} \mathrm{p} / h l)$ \\
\hline FK & $6,3141.10^{-5}$ & $3,7385.10^{-4}$ \\
\hline PC & $5,4407.10^{-5}$ & $1,0969.10^{-5}$ \\
\hline JM & $2,7463.10^{-8}$ & $1,0164.10^{-7}$ \\
\hline
\end{tabular}

Tabela 1: Variâncias dos erros médios quadráticos das variáveis empregando filtro de Kalman (FK), preditor-corretor (PC) e janela móvel (JM) com $\mathrm{H}=250 \mathrm{~s}$.

\section{Conclusões}

Nesse trabalho foram avaliadas três técnicas para a reconciliação de dados, o filtro de Kalman, um algoritmo baseado em um preditor-corretor e o uso de uma janela móvel, que apresentam um baixo custo computacional e uma boa qualidade na reconciliação dos dados. Estas técnicas foram aplicadas a duas variáveis do vaso separador, a pressão de gás $P$ e o nível da fase oleosa na câmara de óleo $h l$. Com base nos resultados obtidos, verificamos que as três técnicas apresentaram um bom resultado na reconciliação dos dados analisados do vaso separador. O método utilizado para comparação de algoritmos foi o do erro médio quadrático entre as variáveis reconciliadas e as variáveis originais, para um dado nível de degradação das variáveis pelo ruído. Através do mesmo, verificamos que o uso de uma janela móvel apresentou o melhor resultado dentre as três técnicas utilizadas.

O presente trabalho pode ser complementado pela análise de algoritmos otimizados em não linearidades, como pertubações, a exemplo do filtro de Kalman estendido. Outra sugestão para um trabalho futuro é a utilização de técnicas de tratamento de erros grosseiros visto que, na ocorrência 
destes, a reconciliação de dados torna-se ineficaz. Essas técnicas podem ser implementadas antes da reconciliação de dados, como prevenção da aplicação desta na presença de erros grosseiros, ou utilizando estatística robusta, em que a reconciliação de dados e o tratamento de erros grosseiros são realizados simultaneamente.

\section{Agradecimentos}

À Coordenação de Pesquisa - COPES da Universidade Federal de Sergipe - UFS pelo financiamento de bolsa de pesquisa e ao prof. Oscar Alberto Zanabria Sotomayor, do Departamento de Engenharia Elétrica - DEL da UFS, pelas contribuições ao trabalho.

\section{Referências}

Albuquerque, J. S. and Biegler, L. T. (1996). Data reconciliation and gross-error detection for dynamic systems, AIChE Journal 42(10): 2841-2856.

Bagajewicz, M. J. and Jiang, Q. (1997). Integral approach to plant linear dynamic reconciliation, AIChE Journal 43(10): 2546-2558.

Bai, S. and Thibault, J. (2010). Dynamic Data Reconciliation: Theory and Practice, Verlag, [S.l.].

Bai, S., Thibault, J. and McLean, D. D. (2006). Dynamic data reconciliation: Alternative to kalman filter, Journal of Process Control 16(5): 485-498.

Benqlilou, C. (2004). Data reconciliation as a framework for chemical processes optimization and control, $\mathrm{PhD}$ thesis, Universitat Politècnica de Catalunya.

Crowe, C. M. (1996). Data reconciliation - progress and challenges, Journal of Process Control 6(2): 89-98.

Darouch, M. and Zasadzinski, M. (1991). Data reconciliation in generalized linear dynamic systems, AIChE Journal 2(37): 193-201.

de Oliveira Azevedo JÚnior, D. (2009). Controle de golfadas em processos de tratamento primário de petróleo, Technical report, Universidade Federal de Sergipe.

Derya B. Özyurt, R. W. P. (2004). Theory and practice of simultaneous data reconciliation and gross error detection for chemical processes, Computers \& Chemical Engineering 28(3): 381-402.
Farias, A. C. (2009). Avaliação de estratégias para reconciliação de dados e detecção de erros grosseiros, Master's thesis, Universidade Federal do Rio Grande Do Sul.

Freire, E. O., Carvalho, E. A. N., Cardoso, C. A. V. and Luciano, B. A. (2008). Execution monitoring applied to data estimation processes, Latin American Robotics Symposium (JRI 2008 - EnRI-LARS2008), Salvador, BA, Brasil.

Gertler, J. and Almasy, G. A. (1973). Balance calculation through dynamic system modeling, Automatica 9: 79-85.

Jang, S., Joseph, B. and Mukai, H. (1986). Comparison of two approaches to on-line parameter and state estimation of nonlinear systems, Industrial Enginnering and Chemical Process Design Development 25: 809-814.

Kuhen, D. R. and Davidson, H. (1961). Computer control. ii. mathematics of control, Chemical Engineering Progress 57: 44-47.

Lawrence, P. J. (1989). Data reconciliation: Getting better information, Hydrocarbon Processing 68: 55-60.

Liebman, M. J., Edgar, T. F. and Lasdon, L. S. (1992). Efficient data reconciliation and estimation for dynamic processes using nonlinear programming techniques, Computers and Chemical Engineering 16: 963-986. DOI: 10.1016/0098-1354(92)80030-D

Mah, R. S., Stanley, G. M. and Downing, D. M. (1976). Reconciliation and rectification of process flow and inventory data, Industrial Engineering and Chemical Process Design Development 15(1): 175-183. DOI: 10.1021/i260057a030

Morad, K., Young, B. R. and Svrcek, W. Y. (2005). Rectification of plant measuremrnts using a statical framework, Computers \&6 Chemical Engineering 29(5): 919-940. DOI: 10.1016/j.compchemeng.2004.07.019

Nunes, G. C., de Medeiros, J. L. and de Queiroz Fernandes AraÚjo, O. (2010). Modelagem e Controle da Produção de Petróleo, Blucher, New York.

Prata, D. M. (2009). Reconciliação Robusta de Dados Para Monitoramento em Tempo Real, $\mathrm{PhD}$ thesis, UFRJ/COPPE.

Stanley, G. and Mah, R. (1977). Estimation of flows and temperatures in process networks, AIChE Journal 23: 642-650. DOI: 10.1002/aic.690230504

Tjoa, I. B. and Biegler, L. T. (1991). Simultaneous strategies for data reconciliation and gross error detection of nonlinear systems, Computers \& Chemical Engineering 15(10): 679690. DOI: 10.1016/0098-1354(91)85014-L 